\title{
Based on network pharmacology and molecular docking to explore the Traditional Chinese Medicine anti-influenza mechanisms:Chinese protocol for diagnosis and treatment of influenza.
}

yifei Chen ${ }^{1}$

${ }^{1}$ Affiliation not available

November 18, 2020

\begin{abstract}
Background Explore the possible mechanism of anti-influenza virus, based on the study of the active components-drug-target network, Protein-Protein Interaction (PPI) network and molecular docking verification, we explored the potential action mechanism of TCM in Chinese protocol for diagnosis and treatment of influenza 2019. Methods Screening the active components and potential targets of 12 drugs in the scheme by using TCMSP database, and Obtaining the target of influenza by GeneCard, Durgbank, OMIM, TTD and PharmGkb databases. Then, constructed the "component-durg-target" network and PPI network were by Cytoscape3.8.0 software. Morethan, analyzed and the biological function and pathway, verified the molecular docking by AutoDock Vina software. Results The 12 drugs in the recommended scheme (XBCQ) for severe influenza contain 192 active components and involve 31 key antiviral targets, which may play an antiviral role through biological processes such as lipopolysaccharide, pathogen molecular reaction and regulate signaling pathway via the IL-17, influenza A, TNF, Toll-like receptors. Conclusion TCM play critical therapeutic roles through "multi-components, multi-targets and multi-pathways" mechanisms in influenza infection. The antiviral pharmacological mechanism of Xuanbai Chengqi decoction, which was analyzed by network pharmacology and molecular docking, provide a new idea for further exploring the diagnosis and treatment of severe influenza.
\end{abstract}

Based on network pharmacology and molecular docking to explore the Traditional Chinese Medicine antiinfluenza mechanisms: Chinese protocol for diagnosis and treatment of influenza.

Key words: Xuanbai Chengqi decoction (XBCQ); Influenza virus; severe Influenza; Network Pharmacology; molecular docking; drug target

Chen yifei1,2\#, Zhou le3\#, Zhu jianjun1, Zhu jianliang1, Huang yao3, Li haojie2, Liu lijun1*

1. Department of Emergency medicine, The Second Affiliated Hospital of Soochow University, Suzhou 215004, China.

2. Department of Emergency medicine, The Affiliated Hospital of Yangzhou University, Yangzhou 225012, China.

3. Yangzhou Center for Disease Control and Prevention, Yangzhou, 225001, China.

\#Contributed equally.

*Corresponding author.

\section{Abstract}




\section{Background}

Explore the possible mechanism of anti-influenza virus, based on the study of the active components-drugtarget network, Protein-Protein Interaction (PPI) network and molecular docking verification, we explored the potential action mechanism of TCM in Chinese protocol for diagnosis and treatment of influenza 2019.

\section{Methods}

Screening the active components and potential targets of 12 drugs in the scheme by using TCMSP database, and Obtaining the target of influenza by GeneCard, Durgbank, OMIM, TTD and PharmGkb databases. Then, constructed the "component-durg-target" network and PPI network were by Cytoscape3.8.0 software. Morethan, analyzed and the biological function and pathway, verified the molecular docking by AutoDock Vina software.

\section{Results}

The 12 drugs in the recommended scheme (XBCQ) for severe influenza contain 192 active components and involve 31 key antiviral targets, which may play an antiviral role through biological processes such as lipopolysaccharide, pathogen molecular reaction and regulate signaling pathway via the IL-17, influenza A, TNF, Toll-like receptors.

\section{Conclusion}

TCM play critical therapeutic roles through "multi-components, multi-targets and multi-pathways" mechanisms in influenza infection. The antiviral pharmacological mechanism of Xuanbai Chengqi decoction, which was analyzed by network pharmacology and molecular docking, provide a new idea for further exploring the diagnosis and treatment of severe influenza.

Epidemics of influenza typically occur during the winter months and have become a public health event that threatens the world. According to the epidemiological model, influenza-associated respiratory mortality are higher than previously WHO stated, about 291 243-645 832 deaths annually [1]. China government attaches great importance to the prevention and treatment of influenza, and the National Health and Health Commission has issued three versions of the Influenza diagnosis and treatment to promote the prevention and treatment of influenza in recent years [2]. In this scheme, in addition to updating antiviral western drugs, severe influenza patients are recommended for treatment on the basis of Xuanbai Chengqi decoction

based on syndrome differentiation. Xuanbai Chengqi decoction (XBCQ) originates from "differentiation of febrile Diseases", The prescription is composed of ephedra, raw gypsum, almond, Anemarrhena anemarrhena, Herba Houttuyniae, Cymbals, Radix Scutellariae, Fritillaria thunbergii, rhubarb, Radix Paeoniae Rubra and raw licorice.

TCM has been used in Asia and even all over the world. However, because of its complex composition and changeable prescription, it is relatively difficult to verify its mechanism by traditional experimental methods. Therefore, there is an urgent need for new methods to systematically and comprehensively analyze the mechanism of Chinese herbal medicine. With the rapid development of bioinformatics, network pharmacology has become a new way to effectively reveal the molecular and pharmacological mechanisms of TCM formulae, and it will also become a new paradigm for TCM research [3-7]. The network pharmacological studies on the anti-influenza effects of compound Artemisia annua, Jingyin granule and Reduning injection have been carried out [8-10].

Molecular docking is an effective and intelligent computational technique to estimate the binding affinity of a ligand (such as drug candidate) in the macromolecular target site (receptor). Based on the structure of active components of TCM and disease targets are clearly, molecular docking is a promising way to show the mechanism of drug action $[11,12]$.

In order to obtain more accurate anti-influenza mechanisms of TCM formulae, we studied the scheme of TCM recommended by the China National Health Commission for severe influenza patients and combined with the methods of network pharmacology and molecular docking to provide a theoretical basis for the prevention 
and control of influenza with TCM. The findings presented in this thesis add to our understanding of TCM for influenza.

1. Materials and methods

1.1. Active ingredients and ingredient targets collection

In Chinese medicine systematic pharmacology database and analysis platform TCMSP (http://lsp.nwu.edu.cn/tcmsp.php), all of the chemical compositions of herbs were available. In order to obtain the active ingredients of the scheme, we screening active ingredients based on absorption, distribution, metabolism and excretion (ADME) parameters. oral bioavailability (OB)[?]30\% and druglikeness (DL)[?] 0.18 are essential for drug screening. Targets corresponding to active components were queried in TCMSP, then, converted target proteins into corresponding Gene names in UniProt database.

\subsection{Acquisition for Influenza disease targets}

Used Drugbank (https://www.drugbank.ca/) and GeneCard (https://www.genecards.org/), OMIM (https://omim.org/), TTD (http://db.idrblab.net/ttd/), PharmGkb (https://www.pharmgkb.org/) database and entered the keyword "Influenza" to search for influenza-related targets. The intersection of the active ingredient targets and the influenza related targets were the key antiviral targets of the active ingredients.

1.3. Network construction and analysis of "active ingredients-key targets"

Active ingredients and key targets were imported to software Cytoscape 3.8.0 and constructed the "active ingredients-key targets" Network, and the "Network Analyzer" function in Cytoscape was used for Network analysis, and the important antivirus components were screened according to the Degree values.

\subsection{Protein-protein interaction (PPI) Network construction and analysis}

We imported the key protein into String database, defined species as human, and set confidence value[?]0.9. The text data after organizing was imported into Cytoscape3.8.0 software to construct PPI Network, and then applied "Network Analyzer" function to analyze the index of Network topology, screened important targets whose degree values were higher than average.

\subsection{Biological function and pathway analysis}

The ClueGO plug-in in Cytoscape3.8.0 software was used to perform GO biological function annotation and KEGG pathway analysis on key targets and set $\mathrm{P}[?] 0$. 05 .

\subsection{Molecular docking Verification}

AutoDock Vina molecular docking software could be used to predict the possible molecular interaction between a target protein and a small molecule. According to the important components in the "Active ingredients - Key targets" network and the durg target proteins in the PPI network, the docking effect between the active ingredients and the key targets was evaluated by AutoDock Vina molecular docking verification.

\section{Results}

\subsection{Drug and disease-related genes}

We obtained 192 active components of 12 herbs (except gypsum) from TCMSP. And then we got 242 targets of active ingredients (Table 1) and 229 influenza-related targets (Figure 1). 31 key targets (figure 2) were obtained after the duplicative terms deleting by Venn diagram.

\subsection{Construction and Analysis of "Active Ingredients - Key Targets" Network}

Network diagram (Figure 3) of antiviral "Active ingredients - Key targets" was constructed in Cytoscape 3.8.0 software with 192 active ingredients corresponding to 12 traditional Chinese medicines and 31 targets (Table 
2). The network diagram involves 188 nodes and 364 edges, among which the circle represents the active ingredients and the rectangle represents the key target points. In terms of degree evaluation, 10 important ingredients were screened, including quercetin, luteolin, kaempferol, wogonin, aloe-emodin, naringenin and etc. The ingredients with high degree values may be the key antivirus ingredients (Table 3 ).

\subsection{PPI network construction and analysis}

31 key target proteins were imported to String database, defined species as human, set confidence value[?]0.9, and PPI network (figure 4) was built in Cytoscape software. The network involves 24 nodes which represent targets and 61 sides which represent interaction between proteins and proteins. And the sizes and colors of the nodes express Degree values. Degree values grow from small to large, so do the nodes. Degree values of 11 target proteins are higher than the average, among which, JUN, CASP8, MAPK1, IL1B's degree values are relatively high, which indicates a strong interaction with other proteins. Therefore, these predictive proteins play an important role in the network.

\subsection{Biological function and pathway analysis}

In Cytoscape 3.8.0 software, ClueGO plug-in was applied to GO biological function annotation and KEGG pathway analysis on 31 key targets. The bar chart of visual analysis was obtained after setting P[?]0.05. As shown in Figure 5, GO analysis revealed that they were significantly enriched in the following biological processes, including response to lipapolysaccharide, molecule of bacterial origin, tumor necrosis factor. Cellular component analysis showed that membrane raft, membrane microdomain and membrane region were common classifications. In terms of molecular functions, they were mainly associated with cytokine receptor binding, cytokine activity, receptor ligand activity, signaling receptor activator. In addition, the pathways enrichment terms were shown by KEGG database. As shown in Figure 6, KEGG pathways included IL17 signailing pathway, hepatitis B, influenza A, Chagas disease, TNF signaling pathway, toll-like recptor signaling pathway.

\subsection{Molecular docking verification}

We selected ingredients with rich contents such as quercetin, luteolin, naringin, and the first four key targets JUN, CASP8, MAPK1 and IL-1B in PPI network. Targets crystal structure files were downloaded from PDB database, and water molecules and ligand molecules were removed by Pymol software. From the Protein Data Bank (PDB) database, 1JNM, 1QDU, 5LCK and 9ILB were identified as the protein structures of the four key targets highlighted above for molecular docking experiments. The Grid Box parameters in AutoDockTools were set as following: JUN, grid center 4040 40, NPTS 10.20 .5 19.5, spacing 1.0; IL1B, grid center -15.8 13.5 -1.6, number of points in xyz (NPTS) 4040 40, spacing 1.0. CASP8 and MAPK1 are dimers and trimers, their active pockets are highly conserved. The docking conditions were similar after 20 times docking and binding energy was used as an important criterion for constituents screening (Table 4). Then we uploaded the structure files of target proteins and quercetin, luteolin, naringin respectively in AutoDock Vina online software. The molecular docking results are shown in Table 4. The closer the protein binds to small molecules, the lower the Full Fitness values are. The results show that the three components can dock with target proteins JUN and IL-1b (Figure 7).

\section{Disscousion}

Influenza is an acute respiratory infectious disease that threatens human beings, which does great harm to high-risk groups in the epidemic season, so it is of great significance to protect severe influenza high-risk groups and actively treat critical influenza patients. In addition to active immunity, the action mechanism of western medicine has been relatively clear, such as oseltamivir, zanamivir and Arbidol. Traditional Chinese medicine is a cultural treasure of Chinese medicine, which has played an important role in the prevention and treatment of influenza in recent years. However, due to the theory of syndrome differentiation of TCM and human treatment, the treatment of TCM is complicated. With the improvement of the separation and extraction technology of monomer components of TCM, the effective components of TCM are gradually defined, supplemented by the deepening of basic research of TCM and the development of biological infor- 
mation. The application of network pharmacology provides a new method for us to predict the potential targets of the effective components of TCM.

Our study shows that the first five active ingredients of XBCQ are quercetin, luteolin, kaempferol, wogonin and aloe emodin. Among the top five active components of Jingyin granulep [13], four coincide with them, and two overlap with the first five of compound Yizhihao [14], which indirectly shows that the three groups have much in common in antiviral active ingredients. The basic research of its monomer against influenza virus has been verified at the different cell types and animal model.

Quercetin is a kind of polyphenols derived from plants, which has anti-inflammatory, anti-viral and anticancer activities [15-17]. Theremore, protein-chemical interactions suggest quercetin as promising drug candidates against COVID-19 as well as other SARS-like viral infections [18]. A strong relationship between quercetin and influenza has been reported in the literature. $\mathrm{Wu} \mathrm{W}$ found that quercetin a broad-spectrum inhibitor of viral infection, including H1N1, H3N2, H5N1.Quercetin interaction with the HA2 subunit and inhibit the entry of the H5N1 virus, which indicates that quercetin acts as an inhibitory in the early stage of infection [19]. These results corroborate the findings of a great deal of the previous work in quercetin against influenza.

Luteolin is a common flavonoid, ranging from scavenging free radicals, anticancer activity to anti-influenza activity. there study show that luteolin is most effective inhibitor to influenza via establishment and verification of a screening method based on AlphaScreen technique[20].Yan $\mathrm{H}$ found that luteolin inhibits the expression of coat protein I complex, which is related to the entry and endocytosis pathway of influenza virus. Its antiviral effect is consistent with previous studies, indicating that the antiviral effect of the effective ingredient of the prescription has a certain theoretical basis [21]. Luteolin inhibited the production of TNF- $\alpha$ and IL-6 in a dose-dependent manner, and shortened the half-life of TNF- $\alpha$ and IL-6mRNA [22]. It has also been reported that luteolin significantly reduced the production of intracellular reactive oxygen species induced by TNF- $\alpha$ in a dose-dependent manner [23]. Luteolin affects the MAPK pathway, which can be demonstrated by the observation of IL-1 $\beta$-induced inhibition of cJunN terminal kinase and p38 kinase activation in SW982 cells. Luteolin treatment also inhibited IL-1 $\beta$-induced AP-1 nuclear translocation [24]. Luteolin also protects mice from lipopolysaccharide-induced acute lung injury by inhibiting the expression of TNF- $\alpha$, IL-6, and COX-2. Luteolin down-regulates LPS-induced leukocyte influx into alveolar space [25]. It was found that luteolin attenuated LPS-induced acute lung injury by inhibiting NF-KB and MAPK pathways $[26]$.

In the protein interaction network, the key gene JUN is a transcription factor which is essential for cellular growth and proliferation, across the cell cycle, self-renewal, metabolism and survival processes. Here, Studies have demonstrated that JUN contributes to fibrotic disease not only by increasing activity through profibrotic programs, but also by influencing the host's overall immune response [27, 28]. Promotes activity of NR5A1 when phosphorylated by HIPK3 would increas steroidogenic gene expression upon cAMP signaling pathway [29]. CASP8 is related to many human diseases. It produces a variety of transcripts and has some non-apoptotic functions, such as regulating the proliferation and differentiation of NK cells and B cells [30]. CASP8 is not only a necessary protease for the exogenous pathway of apoptosis, but also a negative regulator of necrosis [31]. Interleukin-1 $\beta$ (IL-1 $\beta$ ) is an important immune cytokine responsible for inflammation, such as influenza or coinfection bacterica. It is related to the severity of infection disease. All patients with H1N1 infection significantly higher levels of IL-1 $\beta$ and IL-6 than lower respiratory tract infections (LRTI), H1N1 virus infection induces significant upregulation of both interleukins IL1 $\beta$ and IL-6 plasma expressions.[32] IL-1 $\beta$ and interleukin-17A (IL-17A) are key mediators of neutrophilic inflammation in influenza-induced chronic lung inflammation. Blocking of IL-17A or IL-1 resulted to a virtual abrogation of neutrophil recruitment in the initial phase of infection. IL-17A and IL-1 $\beta$ are potential targets for treatment of viral exacerbations [33]. Previous studies have demonstrated that NLRP3 inflammasome regulated IL-1 $\beta$ activation during coinfection, which increases the amount of pro-IL-1 $\beta$ substrate for the inflammasome to process [34]. There KEY Targets responses to lipapolysaccharide, molecule of bacterial origin, tumor necrosis factor biological processes. The small-molecule anti-influenza therapeutic durgs be classified four-category 
currently: primary, neuraminidase inhibitors oseltamivir and zanamivir, which prevent release of nascent virions. Secondary, amantadine and rimantadine, which are M2 ion channel inhibitors. Tertiary, avipirovir and baloxavir, which act on transcription and replication of viral RNA. Quartic, Arbidol has been shown to increase influenza virus HA stability and prevent the low $\mathrm{pH}$-induced HA transition to its fusogenic state. Our study shows that component targets were mainly associated with cytokine receptor binding, cytokine activity, receptor ligand activity, signaling receptor activator in biological processes [35].

The IL-17 family is a subset of cytokines composed of IL-17A-F and plays an important role in both acute and chronic inflammatory responses. Many studies have revealed that delayed clearance of viral load and marked cytokine activation in severe patients of H1N1 virus infection. The IL-17 family signals through its corresponding receptors and activates downstream pathways including NF- $\chi$ B, MAPK and C/EBP to induce the expression of antimicrobial peptides, cytokines and chemokines. In the study of H5N1-infected mice, it was found that IL-17 played a protective role in the process of adaptive immunity of "cytokine storm" and virus clearance [36]. IL-17 has been shown to be protective in severe pandemic influenza, indicating the existence of imbalanced pro-and anti-Th17 responses during this disease [37]. IL-17 deficiency or treatment with monoclonal antibodieswould alleviat acute lung injury induced by the H1N1 virus in mice[38]. In contrast, other groups have showed that IL-17 just played a pathogenic role by recruiting neutrophils to the site of inflammation [39]. IL-17 may act as a 'double-edged sword' and contributes to pulmonary immunopathology [40-42]. The TNF- $\alpha$ and NF- $x \mathrm{~B}$ pathways are central regulators of immune responses, cell survival, and apoptosis, and are modulated by pathogens for their survival. Interference with TNF- $\alpha$ signaling exclusively in distal airway epithelial cells resulted in abrogation of CD8+ mediated lung injury and viral clearance $[43,44]$.

Molecular docking has been used in drug screening of influenza Neuraminidase Inhibitors [45-48]. Our molecular docking studies based on TCM compounds against known protein targets to treatment influenza. Among the four targets chosen for molecular docking experiments, JUN 、 CASP8 - MAPK1 were reported as key gene in signaling pathway mentioned above. Quercetin, luteolin and naringenin were successful docked to JUN target proteins with a high binding energy. It showed that Quercetin, luteolin and naringenin could bind to JUN, and then inhibited influenza through multi-components, multi-targets and multi-pathways. These findings validate the reliability of the active ingredients screened by network pharmacology and their interaction with influenza targets.

\section{Conclusion}

Network pharmacology has gradually become a research method of disease treatment mechanism and drug screening of TCM. It combines system biology, pharmacology, bioinformatics and other multi-disciplinary knowledge, and is a new paradigm of TCM research. Molecular docking technology is an effective supplement to validate candidate drugs. In this study, we analysis the effective components of Xuanbai Chengqi decoction, there potential gene targets against sever influenza and signal pathways potentially, and predicted action mechanismin of TCM by molecular docking. It shows that the antiviral effect of severe influenza with Xuanbai Chengqi decoction plays an anti-influenza role through multi-components, multi-targets and multi-pathways. Season flu coupled with continued COVID-19 outbreaks could increase people's risk of fatal illness. Face to twindemic, Network pharmacology which provided a critical theoretical basis and methods for the treatment of influenza with TCM. We should take all our efforts to share the wealth of TCM and mining the potential therapeutic targets to fight the worldwide epidemic.

\section{Conflicts of Interest}

On behalf of all Authors, the corresponding Author states that there is no conflict of interest related to this study. Each author contributed to the manuscript. Author Contributions: Study concept and design: CYF, ZL,LLJ; Acquisition of data: CYF, ZL, ZJJ, ZJL; Analysis and interpretation of data: HY, LHJ; Drafting of the manuscript: ZJJ; LHJ; Study supervision: LLJ; Revision of the manuscript and approval of the final version of the manuscript: All authors.

\section{Funding}


The study was supported by the Social Development Projects of Yangzhou City (No. YZ2020123).

\section{Reference}

[1] Iuliano AD, Roguski KM, Chang HH, et al; Global Seasonal Influenza-Associated Mortality Collaborator Network.Estimates of global seasonal influenza-associated respiratory mortality: a modelling study. Lancet 2018; 391:1285-300.

[2] National Health Commission of the people's Republic of China, State Administration of Traditional Chinese Medicine. Protocol for diagnosis and treatment of influenza (2019 version). Chinese Journal of Clinical Infectious Diseases, 2019,12 (6): 451-455. DOI: 10.3760/cma.j.issn.1674-2397.2019.06.003

[3] Hopkins AL. Network pharmacology. Nat Biotechnol. 2007 Oct;25(10):1110-1. doi: 10.1038/nbt10071110. PMID: 17921993.

[4] Boezio B, Audouze K, Ducrot P, Taboureau O. Network-based Approaches in Pharmacology. Mol Inform. 2017 Oct;36(10). doi: 10.1002/minf.201700048. Epub 2017 Jul 10. PMID: 28692140.

[5] Hopkins AL. Network pharmacology: the next paradigm in drug discovery. Nat Chem Biol. 2008 Nov;4(11):682-90. doi: 10.1038/nchembio.118. PMID: 18936753.

[6] Li S, Zhang B. Traditional Chinese medicine network pharmacology: theory, methodology and application. Chin J Nat Med. 2013 Mar;11(2):110-20. doi: 10.1016/S1875-5364(13)60037-0. PMID: 23787177.

[7] Lee WY, Lee CY, Kim YS, Kim CE. The Methodological Trends of Traditional Herbal Medicine Employing Network Pharmacology. Biomolecules. 2019 Aug 13;9(8):362. doi: 10.3390/biom9080362. PMID: 31412658; PMCID: PMC6723118.

[8] Liu J, Sun K, Zheng C, Chen X, Zhang W, Wang Z, Shar PA, Xiao W, Wang Y. Pathway as a pharmacological target for herbal medicines: an investigation from reduning injection. PLoS One. 2015 Apr 1;10(4):e0123109. doi: 10.1371/journal.pone.0123109. PMID: 25830385; PMCID: PMC4382287.

[9] Kim M, Park KH, Kim YB. Identifying Active Compounds and Targets of Fritillariae thunbergii against Influenza-Associated Inflammation by Network Pharmacology Analysis and Molecular Docking. Molecules. 2020 Aug 25;25(17):3853. doi: 10.3390/molecules25173853. PMID: 32854331; PMCID: PMC7504253.

[10] Han L, Wei XX, Zheng YJ, Zhang LL, Wang XM, Yang HY, Ma X, Zhao LH, Tong XL. Potential mechanism prediction of Cold-Damp Plague Formula against COVID-19 via network pharmacology analysis and molecular docking. Chin Med. 2020 Jul 30;15:78. doi: 10.1186/s13020-020-00360-8. PMID: 32754224; PMCID: PMC7391051.

[11] Yuriev E, Agostino M, Ramsland PA. Challenges and advances in computational docking: 2009 in review. J Mol Recognit. 2011;24:149-64.

[12] Daina A, Michielin O, Zoete V. Swiss Target Prediction: updated data and new features for efficient prediction of protein targets of small molecules. Nucleic Acids Res. 2019 Jul 2;47(W1):W357-W364. doi: 10.1093/nar/gkz382. PMID: 31106366; PMCID: PMC6602486.

[13] Yu Ting, Liu Xinjuan, Liu Li,Gao Qi. Study on the Mechanism of Jingyin granule in the treatment of viral Cold based on Network Pharmacology. Chinese Traditional Patent Medicine.2020, Feb,42(2):456-461.

[14] Xu Lü-jie, Jiang Wen, Pang Xiao-cong, Kang De, Xiong Wan-di, LIU rui, Xing Jian-guo, Liu Ai-lin, Du Guan-hua. Network pharmacology study of the effective constituents in the Compound Yizhihao against influenza disease. Acta Pharmaceutica Sinica,2017 May,52(5):745-752.

[15]Li Y, Yao J, Han C, Yang J, Chaudhry MT, Wang S, Liu H, Yin Y. Quercetin, Inflammation and Immunity. Nutrients. 2016 Mar 15;8(3):167. doi: 10.3390/nu8030167. PMID: 26999194; PMCID: PMC4808895. 
[16] Kumar P, Sharma S, Khanna M, Raj HG. Effect of Quercetin on lipid peroxidation and changes in lung morphology in experimental influenza virus infection. Int J Exp Pathol. 2003 Jun;84(3):127-33. doi: 10.1046/j.1365-2613.2003.00344.x. PMID: 12974942; PMCID: PMC2517549.

[17] Liu Z, Zhao J, Li W, Shen L, Huang S, Tang J, Duan J, Fang F, Huang Y, Chang H, Chen Z, Zhang R. Computational screen and experimental validation of anti-influenza effects of quercetin and chlorogenic acid from traditional Chinese medicine. Sci Rep. 2016 Jan 12;6:19095. doi: 10.1038/srep19095. PMID: 26754609; PMCID: PMC4709578.

[18] Nain Z, Rana HK, Liò P, Islam SMS, Summers MA, Moni MA. Pathogenetic profiling of COVID-19 and SARS-like viruses. Brief Bioinform. 2020 Aug 11:bbaa173. doi: 10.1093/bib/bbaa173. Epub ahead of print. PMID: 32778874; PMCID: PMC7454314.

[19] Wu W, Li R, Li X, He J, Jiang S, Liu S, Yang J. Quercetin as an Antiviral Agent Inhibits Influenza A Virus (IAV) Entry. Viruses. 2015 Dec 25;8(1):6. doi: 10.3390/v8010006. PMID: 26712783; PMCID: PMC4728566.

[20] Zima V, Radilová K, Kožíšek M, Albiñana CB, Karlukova E, Brynda J, Fanfrlík J, Flieger M, Hodek J, Weber J, Majer P, Konvalinka J, Machara A. Unraveling the anti-influenza effect of flavonoids: Experimental validation of luteolin and its congeners as potent influenza endonuclease inhibitors. Eur J Med Chem. 2020 Aug 22;208:112754. doi: 10.1016/j.ejmech.2020.112754. Epub ahead of print. PMID: 32883638.

[21] Yan H, Ma L, Wang H, Wu S, Huang H, Gu Z, Jiang J, Li Y. Luteolin decreases the yield of influenza A virus in vitro by interfering with the coat protein I complex expression. J Nat Med. 2019 Jun;73(3):487-496. doi: 10.1007/s11418-019-01287-7. Epub 2019 Feb 13. PMID: 30758716.

[22] Wu, W., Li, D., Zong, Y., Zhu, H., Pan, D., Xu, T., Wang, T., Wang, T., 2013. Luteolin inhibits inflammatory responses via p38/MK2/TTP-mediated mRNA stability.Molecules 18, 8083-809.

[23] Xia, F., Wang, C., Jin, Y., Liu, Q., Meng, Q., Liu, K., Sun, H., 2014. Luteolin protects HUVECs from TNF-alpha-induced oxidative stress and inflammation via its effects on the Nox4/ROS-NF-kappaB and MAPK pathways. J. Atheroscler. Thromb. 21,768-783.

[24] Choi, E.M., Lee, Y.S., 2010. Luteolin suppresses IL-1beta-induced cytokines and MMPs production via p38 MAPK, JNK, NF-kappaB and AP-1 activation in human synovial sarcoma cell line, SW982. Food Chem. Toxicol. 48, 2607-2611.

[25] Li, Y.C., Yeh, C.H., Yang, M.L., Kuan, Y.H., 2012. Luteolin suppresses inflammatory mediator expression by blocking the Akt/NFkappaB pathway in acute lung injury induced by lipopolysaccharide in mice. Evid. Based Complement. Altern. Med. 2012,383608.[26] Kuo, M.Y., Liao, M.F., Chen, F.L., Li, Y.C., Yang, M.L., Lin, R.H., Kuan, Y.H., 2011.Luteolin attenuates the pulmonary inflammatory response involves abilities of anti-oxidation and inhibition of MAPK and NFkappaB pathways in mice with endotoxin-induced acute lung injury. Food Chem. Toxicol. 49, 2660-2666.

[27] Qing J, Zhang Y, Derynck R. Structural and functional characterization of the transforming growth factor-beta -induced Smad3/c-Jun transcriptional cooperativity. J Biol Chem. 2000 Dec 8;275(49):38802-12. doi: 10.1074/jbc.M004731200. PMID: 10995748.

[28] Ji Z, Donaldson IJ, Liu J, Hayes A, Zeef LA, Sharrocks AD. The forkhead transcription factor FOXK2 promotes AP-1-mediated transcriptional regulation. Mol Cell Biol. 2012 Jan;32(2):385-98. doi: 10.1128/MCB.05504-11. Epub 2011 Nov 14. Erratum in: Mol Cell Biol. 2014 May;34(10):1892. PMID: 22083952; PMCID: PMC3255788.

[29] Lan HC, Li HJ, Lin G, Lai PY, Chung BC. Cyclic AMP stimulates SF-1-dependent CYP11A1 expression through homeodomain-interacting protein kinase 3-mediated Jun N-terminal kinase and c-Jun phosphorylation. Mol Cell Biol. 2007 Mar;27(6):2027-36. doi: 10.1128/MCB.02253-06. Epub 2007 Jan 8. PMID: 17210646; PMCID: PMC1820514. 
[30] Lin Z, Guo Z, Xu Y, Zhao X. Identification of a secondary promoter of CASP8 and its related transcription factor PUR $\alpha$. Int J Oncol. 2014 Jul;45(1):57-66. doi: 10.3892/ijo.2014.2436. Epub 2014 May 9. PMID: 24819879; PMCID: PMC4079158.

[31] Ghanekar Y, Sadasivam S. In silico analysis reveals a shared immune signature in CASP8-mutated carcinomas with varying correlations to prognosis. PeerJ. 2019 Feb 11;7:e6402. doi: 10.7717/peerj.6402. PMID: 30775178; PMCID: PMC6375258.

[32]Chiaretti A, Pulitanò S, Barone G, Ferrara P, Romano V, Capozzi D, Riccardi R. IL-1 $\beta$ and IL-6 upregulation in children with H1N1 influenza virus infection. Mediators Inflamm. 2013;2013:495848. doi: 10.1155/2013/495848. Epub 2013 Apr 29. PMID: 23737648; PMCID: PMC3657430.

[33] Sichelstiel A, Yadava K, Trompette A, Salami O, Iwakura Y, Nicod LP, Marsland BJ. Targeting IL-1 $\beta$ and IL-17A driven inflammation during influenza-induced exacerbations of chronic lung inflammation. PLoS One. 2014 Jun 11;9(2):e98440. doi: 10.1371/journal.pone.0098440. PMID: 24918427; PMCID: PMC4053370.

[34]Rodriguez AE, Bogart C, Gilbert CM, McCullers JA, Smith AM, Kanneganti TD, Lupfer CR. Enhanced IL-1 $\beta$ production is mediated by a TLR2-MYD88-NLRP3 signaling axis during coinfection with influenza A virus and Streptococcus pneumoniae. PLoS One. 2019 Feb 22;14(2):e0212236. doi: 10.1371/journal.pone.0212236. PMID: 30794604; PMCID: PMC6386446.

[35] Uyeki TM, Bernstein HH, Bradley JS, Englund JA, File TM, Fry AM, Gravenstein S, Hayden FG, Harper SA, Hirshon JM, Ison MG, Johnston BL, Knight SL, McGeer A, Riley LE, Wolfe CR, Alexander PE, Pavia AT. Clinical Practice Guidelines by the Infectious Diseases Society of America: 2018 Update on Diagnosis, Treatment, Chemoprophylaxis, and Institutional Outbreak Management of Seasonal Influenzaa. Clin Infect Dis. 2019 Mar 5;68(6):e1-e47. doi: 10.1093/cid/ciy866. Erratum in: Clin Infect Dis. 2019 May 2;68(10):1790. PMID: 30566567; PMCID: PMC6653685.

[36]To KK, Hung IF, Li IW, Lee KL, Koo CK, Yan WW, Liu R, Ho KY, Chu KH, Watt CL, Luk WK, Lai KY, Chow FL, Mok T, Buckley T, Chan JF, Wong SS, Zheng B, Chen H, Lau CC, Tse H, Cheng VC, Chan $\mathrm{KH}$, Yuen KY. Delayed clearance of viral load and marked cytokine activation in severe cases of pandemic H1N1 2009 influenza virus infection. Clin Infect Dis. 2010;50:850-859. doi: 10.1086/650581.

[37]Li, C., Yang, P., Sun, Y., Li, T., Wang, C., Wang, Z., Zou, Z., Yan, Y., Wang, W., Wang, C., Chen, Z., Xing, L., Tang, C., Ju, X., Guo, F., Deng, J., Zhao, Y., Yang, P., Tang, J., Wang, H., .. Jiang, C. (2012). IL-17 response mediates acute lung injury induced by the 2009 pandemic influenza A (H1N1) virus. Cell research, 22(3), 528-538. https://doi.org/10.1038/cr.2011.165

[38]Wang X, Chan CC, Yang M, Deng J, Poon VK, Leung VH, Ko KH, Zhou J, Yuen KY, Zheng BJ, Lu L. A critical role of IL-17 in modulating the B-cell response during H5N1 influenza virus infection. Cell Mol Immunol. 2011 Nov;8(6):462-8. doi: 10.1038/cmi.2011.38. Epub 2011 Sep 26. PMID: 21946434; PMCID: PMC4012931.

[39] Almansa R, Socias L, Ramirez P, Martin-Loeches I, Vallés J, Loza A, Rello J, Kelvin DJ, León C, Blanco J, Andaluz D, Micheloud D, Maraví E, Ortiz de Lejarazu R, Bermejo-Martin JF. Imbalanced pro- and anti-Th17 responses (IL-17/granulocyte colony-stimulating factor) predict fatal outcome in 2009 pandemic influenza. Crit Care. 2011;15(5):448. doi: 10.1186/cc10426. Epub 2011 Oct 20. PMID: 22040730; PMCID: PMC3334743.

[40]Park H, Li Z, Yang XO, Chang SH, Nurieva R, Wang YH, et al. A distinct lineage of CD4 T cells regulates tissue inflammation by producing interleukin 17. Nat Immunol. 2005;6:1133-1141.

[41] Pelletier M, Maggi L, Micheletti A, Lazzeri E, Tamassia N, Costantini C, Cosmi L, Lunardi C, Annunziato F, Romagnani S, Cassatella MA. Evidence for a cross-talk between human neutrophils and Th17 cells. Blood. 2010 Jan 14;115(2):335-43. doi: 10.1182/blood-2009-04-216085. Epub 2009 Nov 4. PMID: 19890092. 
[42] Lindén A, Laan M, Anderson GP. Neutrophils, interleukin-17A and lung disease. Eur Respir J. 2005 Jan;25(1):159-72. doi: 10.1183/09031936.04.00032904. PMID: 15640338.

[43]Srikiatkhachorn A, Chintapalli J, Liu J, Jamaluddin M, Harrod KS, Whitsett JA, Enelow RI, Ramana CV. Interference with intraepithelial TNF- $\alpha$ signaling inhibits CD8(+) T-cell-mediated lung injury in influenza infection. Viral Immunol. 2010 Dec;23(6):639-45. doi: 10.1089/vim.2010.0076. PMID: 21142450; PMCID: PMC2991178.

[44]Aldridge JR Jr, Moseley CE, Boltz DA, Negovetich NJ, Reynolds C, Franks J, Brown SA, Doherty PC, Webster RG, Thomas PG. TNF/iNOS-producing dendritic cells are the necessary evil of lethal influenza virus infection. Proc Natl Acad Sci U S A. 2009 Mar 31;106(13):5306-11. doi: 10.1073/pnas.0900655106. Epub 2009 Mar 11. PMID: 19279209; PMCID: PMC2664048.

[45] Salmaso V, Moro S. Bridging Molecular Docking to Molecular Dynamics in Exploring Ligand-Protein Recognition Process: An Overview. Front Pharmacol. 2018 Aug 22;9:923. doi: 10.3389/fphar.2018.00923. PMID: 30186166; PMCID: PMC6113859.

[46] Lindström A, Edvinsson L, Johansson A, Andersson CD, Andersson IE, Raubacher F, Linusson A. Postprocessing of docked protein-ligand complexes using implicit solvation models. J Chem Inf Model. 2011 Feb 28;51(2):267-82. doi: 10.1021/ci100354x. Epub 2011 Feb 10. PMID: 21309544.

[47]Lu SJ, Chong FC. Combining molecular docking and molecular dynamics to predict the binding modes of flavonoid derivatives with the neuraminidase of the $2009 \mathrm{H} 1 \mathrm{~N} 1$ influenza A virus. Int J Mol Sci. 2012;13(4):4496-507. doi: 10.3390/ijms13044496. Epub 2012 Apr 10. PMID: 22605992; PMCID: PMC3344228.

[48]Inamdar P, Bhandari S, Sonawane B, Hole A, Jadhav C. Structure Optimization of Neuraminidase Inhibitors as Potential Anti-Influenza (H1N1Inhibitors) Agents Using QSAR and Molecular Docking Studies. Iran J Pharm Res. 2014 Winter;13(1):49-65. PMID: 24734056; PMCID: PMC3985258.

\section{Figure Legends}

Fig1 Venn diagrams for influenza target. Based on Drugbank, GeneCard, OMIM, TTD, PharmGkbdatabase, 229 influenza-related targets were found.

Fig2 Venn diagrams for the co-target between 242 targets of active ingredients and 229 influenza-related targets. 31 key targets were obtained after the duplicative terms deleted.

Fig3. Network diagram between active components and target genes. The circle hexagon nodes represent the active components, the blue hexagon nodes represent the target genes. Nodes size are proportional to their degree.

Fig4. PPI network construction and analysis. PPI network of the co-expressed genes. The PPI network was constructed via the Search Tool for the Retrieval of Interacting Genes online, degree values of 11 target proteins are higher than the average for further analysis.

Fig5.Top 10 GO enrichments in BP,CC,MF (Fig 5) GO enrichment. X-axis is enrichment gene ratio, Y-axis is biological process, molecular function and cellular component analysis. Color represents the adjusted p-value, the bluer the color, the smaller the adjusted p-value.

Fig6. KEGG pathway enrichment and top 30 KEGG pathways annotation. (Fig 6). X-axis is enrichment gene count, Y-axis is KEGG pathway, and the color of bar chart represents the adjusted p-value.

Fig7.The docking complex of four targets and their strongest binding components. the active site residues are shown. JUN(7A-7C). ILIB (7D-7F).

\section{Hosted file}


Supplementary figure.pdf available at https://authorea.com/users/376775/articles/493653based-on-network-pharmacology-and-molecular-docking-to-explore-the-traditional-chinesemedicine-anti-influenza-mechanisms-chinese-protocol-for-diagnosis-and-treatment-ofinfluenza

\section{Hosted file}

table.pdf available at https://authorea.com/users/376775/articles/493653-based-on-networkpharmacology-and-molecular-docking-to-explore-the-traditional-chinese-medicine-antiinfluenza-mechanisms-chinese-protocol-for-diagnosis-and-treatment-of-influenza 